\title{
Myocardial Contrast Agents - Safety Considerations And Clinical Efficacy In Stress Echocardiography
}

${ }^{1}$ University of Medicine and Pharmacy Tirgu Mures

\begin{abstract}
Transthoracic echocardiographic examination is known to be a safe, non-invasive and reproducible method, used in every day clinical practice to obtain important information about cardiac structure and function. Unfortunately, a significant proportion of studies have highlighted the considerable technically difficultly in producing diagnostic images due to a poor acoustic window and more than $33 \%$ of patients undergoing stress echocardiography have suboptimal echocardiographic images. All these limitations have led to the use of contrast agents to improve the quality of standard ultrasound examination to provide a better delineation of left ventricle endocardial borders or to obtain information that cannot be achieved by using standard echocardiography, such as assessing myocardial microcirculation and therefore perfusion. This paper sought to review the clinical efficacy and safety of ultrasound contrast agents focusing on stress echocardiography.
\end{abstract}

Keywords: contrast agent, safety, stress echocardiography

\section{Maria Mihaela Opris}

University of Medicine and Pharmacy Tirgu Mures

Str. Gheorghe Marinescu, nr. 38

email : drmihaelaopris@gmail.com

phone: +40744626571

\section{Introduction}

Transthoracic echocardiographic examination is known to be a safe, non-invasive and reproducible method, used in every day clinical practice to obtain important information about cardiac structure and function. Unfortunately, a significant proportion of studies have highlighted the considerable technically difficultly in producing diagnostic images due to a poor acoustic window, particularly in patients with chest wall deformities, obesity and lung disease, or in critically ill patients with mechanical ventilation where echocardiography provides important data that can immediately change the patient's management [1-3]. More than 33\% of patients undergoing stress echocardiography have suboptimal echocardiographic images and even more importantly in patients with significant left ventricular (LV) dysfunction [4]. All these limitation have led to the use of contrast agents to improve the quality of standard ultrasound examination to provide a better delineation of LV endocardial borders or to obtain information that cannot be achieved by using standard echocardiography, such as assessing myocardial microcirculation and therefore perfusion [5]. This paper sought to review the clinical efficacy and safety of ultrasound contrast agents focusing on 
stress echocardiography.

\section{Contrast agents}

In ultrasound contrast echocardiography, the contrast agent currently used is acoustically active gas filled microbubbles with a protein, lipid or polymer stabilising shell and a diameter smaller than red blood cells [6], administered by intravenous injection as a bolus or as a continuous infusion, remaining confined within the intravascular space and passing through the pulmonary capillary bed. Microbubblebased agents are isotonic to human plasma, and after the microbubbles are destroyed, the shell is removed by the reticuloendothelial system, while the gas is eliminated through the respiratory system [7].

The earliest references to contrast agents in ultrasound imaging dates from 1968, when Gramiak and Shah first introduced the idea of injecting agitated saline into the supravalvular aorta during echocardiographic examinations, producing large and unstable microbubbles, spreading into solutions in less than 10 seconds [8]. Over the years several contrast agents have been studied; the first generation was highly unstable, dissolved rapidly in blood and lost their echogenicity. Because of their large diameter, larger than the capillary lumen, a significant amount of microbubbles were filtered by the lungs, while air-filled bubbles smaller than red blood cells $(<7 \mu \mathrm{m})$ were able to pass through pulmonary microcirculation but collapsed in a few seconds due to surface tension and surrounding blood pressure, resulting in gas diffusion from the microbubbles into the blood $[9 ; 10]$. The first microbubble-based contrast agent commercially available in Europe in 1991 approved for echocardiography to detect cardiac shunts was Echovist (Schering, Berlin, Germany), but the air-filled microbubbles stabilised within a galactose matrix corresponding to the peripheral shell were not stable enough to pass through the lungs after a peripheral intravenous injection [7]. To overcome these limitations two major changes were made. First
Albunex (Mallinckrodt, St. Louis USA) was produced by sonicating $5 \%$ of human albumin to obtain a protein shell to stabilise the air-filled microbubbles [11] and because of their sensitivity to pressure changes and their very short half-life $(<1 \mathrm{~min})$ it is no longer in production [7]. Then a fatty acid - palmitic acid - was used to cover the air-filled microbubbles of the agent Levovist (Schering AG, Berlin, Germany) to reduce surface tension in order to increase the stability and to allow for multiple recirculation (1-5 $\mathrm{min}$ ) [12].

Even so things were far from what would be an ideal contrast agent, meaning an inert, stable during transpulmonary passage agent, persisting within the blood or with a well-specified tissue distribution, ensuring the necessary time for imaging examination. Under these conditions, a second generation of contrast agents was needed. The air in the bubble was changed to higher molecular-weight gases, such as perfluorocarbons and sulphur hexafluoride gas, with a low diffusion coefficient and low solubility in blood, persisting longer in the bloodstream, and the shell was design to improve the stability of the microbubbles [7].

A number of ultrasound contrast agents have been developed: EchoGen (Sonus Pharmaceuticals, USA), Optison (GE Healthcare, Princeton, NJ, USA), Definity (marketed in North America as Luminity by Lantheus Medical Imaging, North Billerica, MA, USA), Sonazoid (GE Healthcare, Oslo, Norway), SonoVue (Bracco, Milano, Italy), are some examples of agents known as 'second generation contrast agents'.

EchoGen (Sonus Pharmaceuticals, USA) is a perflenapent liquid emulsion which contains dodecafluropentane liquid in the dispersed phase and shifts to a gas phase at body temperature, creating microbubbles [7; 13]. Despite having received European approval for cardiac use in 2000, it was withdrawn from the market by Sonus Pharmaceuticals.

Optison (GE Healthcare, Princeton, NJ, USA) is a second generation contrast agent consisting of sonicated albumin microspheres filled with octafluoropropane (perflutren) with a mean diameter of $3-4.5 \mu \mathrm{m}$ for the microbubbles $[14 ; 15]$.

Definity (Lantheus Medical Imaging, North Billerica, MA, USA) contains octafluoropropane (perflutren) encapsulated in an outer lipid shell, 
consisting of a single layer of phospholipids and with a medium diameter of $2.5 \mu \mathrm{m}$. It is rapidly cleared from systemic circulation through the lungs, and in most cases after a few minutes (4-5 min) perflutren was undetectable in blood and expired air [16;17].

Sonazoid (GE Healthcare, Oslo, Norway) consists of microbubbles containing perfluorobutane within a hydrogenated egg phosphatidylserine shell, with a well-defined size range and a mean diameter of $3 \mu \mathrm{m}[18]$.

SonoVue (Bracco, Milano, Italy) consists of microbubbles containing sulphur hexafluoride within a flexible phospholipid shell and with a mean diameter of $3 \mu \mathrm{m}$. The low solubility of the gas, the stability of the phospholipid shell and the uniformity of the microbubble diameter provides high and prolonged stability in the blood. After destruction of the microbubbles, more than $80 \%$ of the gas is exhaled through the lungs in $11 \mathrm{~min}$ [19].

\section{Safety considerations}

In 1997, the US Food and Drug Administration (FDA) approved the use of contrast agents to improve the quality of standard ultrasound after considering safety and efficacy data. The studies performed found no significant changes in systemic or pulmonary hemodynamic, myocardial contractility or perfusion [20]. After reporting some adverse events due to contrast agent administration, the FDA and the European Medicines Agency (EMEA) imposed a number of restrictions in cardiac practice. In May 2004, the EMEA temporary suspended the use of Sonovue (Bracco, Milano, Italy) for cardiac indications, based on reports of 3 cases of fatal adverse events and 19 cases of severe, non-fatal adverse events with a temporal relationship to this contrast agent administration in a post-marketing analysis of 157,838 studies. In July 2004, the Committee for Medical Products for Human Use (CHMP) recommended reinstatement for cardiac indication with some restrictions (exclusion of patients with recent acute coronary syndrome or clinically unstable ischaemic cardiac disease) [19]. In October 2007, after a similar post-marketing analysis, the FDA mandated that a 'black box' warning be placed on Definity (Lantheus Medical Imaging, North Billerica, MA, USA) and Optison (GE Healthcare, Princeton, NJ, USA), following reports of postcontrast adverse reactions, including 11 deaths (10 after the administration of Definity and one after Optison) which appeared to be temporally related, but not clearly causally attributable to ultrasound contrast agent administration. Four fatal cases occurred within 30 minutes after administration (one in a ventilated patient with sepsis, two in patients with severe heart failure and one after a stress test) and six cases that occurred up to 12 hours after contrast injection associated with serious underlying conditions [21]. To better define the risk-benefit relationship associated with the use of contrast agents in echocardiographic studies a number of retrospective studies were performed [22-25]. In a retrospective study design to examine the short- and long-term safety profiles associated with the use of ultrasound contrast agents, Dolan et al. analysed 42,408 patients from 3 different medical centres who had baseline suboptimal images and/or underwent myocardial perfusion imaging and received contrast agents; 18,749 underwent stress echocardiography. No deaths or acute myocardial infarction were observed within $30 \mathrm{~min}$; 1 death and 5 nonfatal myocardial infarctions were observed within $24 \mathrm{~h}$. These serious adverse events, occurring within $24 \mathrm{~h}$ after the contrast injection, could be explained by the patient's underlying condition or to induced ischaemia during stress echocardiography. In conclusion the risk of both short-term and long-term events (nonfatal myocardial infarction and death) after contrast administration were very low and the incidence of adverse events was similar in patients who did or did not receive ultrasound contrast agent during stress echocardiography [20]. More recently, in a retrospective observational outcome study design to compare all-cause $48 \mathrm{~h}$ and hospital stay mortality in critically ill patients who underwent echocardiography either with or without ultrasound contrast agent, Main et al. showed an association with a $28 \%$ lower mortality at $48 \mathrm{~h}$ in patients undergoing echocardiography with contrast agent in comparison with patients undergoing non-contrast-enhanced 
echocardiography. Possible explanations for these results could be a better management of patients due to more timely and accurate diagnosis after contrast agent administration and avoidance of other invasive tests [22].

The current FDA labelling is as follows: 'Serious cardiopulmonary reactions, including fatalities, have occurred uncommonly during or following perflutren-containing microsphere administration. Most serious reactions occur within 30 minutes of administration. Assess all patients for the presence of any condition that precludes Definity/ Optison administration. Always have resuscitation equipment and trained personnel readily available'. The current contraindications to contrast are: (1) right-to-left, bidirectional, or transient right-to-left cardiac shunts; (2) hypersensitivity to perflutren; and (3) hypersensitivity to blood, blood products, or albumin (in the case of Optison only) [26]

Although anaphylactoid reactions to ultrasound contrast agents are rare, it is recommended to use a protocol in the echocardiography laboratory for early recognition and effective management of these acute life-threatening reactions [26]. The most severe forms are associated with bronchospasm, shock (hypotension, hypoxemia), and other reactions include urticaria, palmar erythema, back pain (more common with Definity), central nervous reactions: seizures, convulsions and altered consciousness. The main hypothesis for these reactions is related to a complement-mediated idiosyncratic reaction that can also be observed with other liposomal drugs. Lipid particles are likely to produce this $\mathrm{C}$ activation-related pseudoallergy-like syndrome (CARPA). A CARPAlike syndrome can occur, but it is rare $(<1: 10,000)$, and it is unknown if it is causally related to any of the reported deaths, and should not be considered an excuse for not using contrast [27].

Therefore, clinical evidence based on a large patient database showed that contrast echocardiography is a safe and useful diagnostic tool to evaluate cardiac structure and function with a high benefit-to-risk ratio and with less risk than other commonly used contrast agents in other imaging modalities [26].

\section{Contrast stress echocardiography}

The currently available ultrasound systems have pre-programmed settings for control echocardiography that can be adapted in order to optimise the study. A low mechanical index (MI) (0.1-0.3) is recommended to avoid disruption of microbubbles and allows for continuous image acquisition, while a high mechanical index $(>0.5)$ destroys the bubbles [28]. The real-time very low MI techniques permit the enhanced detection of microbubbles within the LV cavity and myocardium, enabling cardiac function and myocardial perfusion information to be obtained at the same time [26;29]. In order to quantify myocardial perfusion, a stable concentration of the contrast agent is required, and after the high mechanical index ultrasound flash to destroy the bubbles, continuous imaging at low mechanical index follows to display the replenishment of the myocardium with microbubbles in real time [29].

Stress echocardiography is a widely used technique for detecting the presence and extent of myocardial ischaemia and myocardial viability in patients with LV dysfunction. Because both myocardial ischaemia and myocardial viability criteria are based on wall motion abnormalities, an accurate visualisation of all walls of the LV is needed [28]. Ultrasound contrast agents produce myocardial opacification and improve the delineation of the endocardial borders, allowing for an accurate assessment of LV volume and function. Wall motion analysis using enhanced LV opacification led to improvements in the sensitivity, specificity, better reproducibility and diagnostic accuracy compared to those who did not require UCAs $[20 ; 30 ; 31 ; 32]$. Ultrasound contrast agents should be used during stress echocardiography whenever suboptimal images exist for the quantification of chamber volumes and ejection fraction as well as the assessment of regional wall motion. Suboptimal images are defined as the inability to detect two or more contiguous segments in any three of the apical windows [26].

Although myocardial perfusion imaging is 
not an approved indication for ultrasound contarst agents, it has been used in multiple clinical studies to examine perfusion to improve the detection of coronary artery disease in the emergency department, improve the detection of coronary artery disease during stress echocardiography and improve the diagnostic evaluation of cardiac masses [26]. Shah et al. showed that the incorporation of myocardial contrast echocardiography into a clinical stress echocardiography service is either of incremental benefit over wall motion (WM) analysis or gives added confidence with WM analysis in the majority of cases [33].

\section{Conclusions}

Contrast use in patients with suboptimal images improves feasibility and accuracy of rest and stress echocardiography, provides additional information on myocardial perfusion, reduces the need for additional alternative imaging techniques and invasive investigation with clear evidence of safe use of contrast agents in echocardiography.

\section{References}

1. Olszewski, R., Timperley, J., Szmigielski, C., Monaghan, M., Nihoyannopoulos, P., Senior, R., \& Becher, H. (2007). The clinical applications of contrast echocardiography. Eur $J$ Echocardiogr, 8(3), S13-23. doi:10.1016/j.euje.2007.03.021

2. Mulvagh, S. L., Rakowski, H., Vannan, M. A., Abdelmoneim, S. S., Becher, H., Bierig, S. M., Burns, P. N., Castello, R., Coon, P. D., Hagen, M. E., Jollis, J. G., Kimball, T.
R., Kitzman, D. W., Kronzon, I., Labovitz, A. J., Lang, R. M., Mathew, J., Moir, W. S., Nagueh, S. F., Pearlman, A. S., Perez, J. E., Porter, T. R., Rosenbloom, J., Strachan, G. M., Thanigaraj, S., Wei, K., Woo, A., Yu, E. H., Zoghbi, W. A., \& American Society of, E. (2008). American Society of Echocardiography Consensus Statement on the Clinical Applications of Ultrasonic Contrast Agents in Echocardiography. $J$ Am Soc Echocardiogr, 21(11), 1179-1201; quiz 1281. doi:10.1016/j.echo.2008.09.009

3. Weissman, N. J., Cohen, M. C., Hack, T. C., Gillam, L. D., Cohen, J. L., \& Kitzman, D. W. (2000). Infusion versus bolus contrast echocardiography: a multicenter, open-label, crossover trial. Am Heart J, 139(3), 399-404.

4. Thanigaraj, S., Nease, R. F., Jr., Schechtman, K. B., Wade, R. L., Loslo, S., \& Perez, J. E. (2001). Use of contrast for image enhancement during stress echocardiography is cost-effective and reduces additional diagnostic testing. Am J Cardiol, 87(12), 1430-1432.

5. Senior, R., Becher, H., Monaghan, M., Agati, L., Zamorano, J., Vanoverschelde, J. L., \& Nihoyannopoulos, P. (2009). Contrast echocardiography: evidence-based recommendations by European Association of Echocardiography. Eur $J$ Echocardiogr, 10(2), 194-212. doi:10.1093/ejechocard/ jep005

6. Mulvagh, S. L., DeMaria, A. N., Feinstein, S. B., Burns, P. N., Kaul, S., Miller, J. G., Monaghan, M., Porter, T. R., Shaw, L. J., \& Villanueva, F. S. (2000). Contrast echocardiography: current and future applications. J Am Soc Echocardiogr, 13(4), 331-342.

7. Quaia, E. (2006). Contrast Media in Ultrasonography: Basic Principles and Clinical Applications: Springer Berlin Heidelberg. 
8. Gramiak, R., \& Shah, P. M. (1968). Echocardiography of the aortic root. Invest Radiol, 3(5), 356-366.

9. Feinstein, S. B., Shah, P. M., Bing, R. J., Meerbaum, S., Corday, E., Chang, B. L., Santillan, G., \& Fujibayashi, Y. (1984). Microbubble dynamics visualized in the intact capillary circulation. $J \mathrm{Am}$ Coll Cardiol, 4(3), 595-600..

10. Shapiro, J. R., Reisner, S. A., Lichtenberg, G. S., \& Meltzer, R. S. (1990). Intravenous contrast echocardiography with use of sonicated albumin in humans: systolic disappearance of left ventricular contrast after transpulmonary transmission. $\mathrm{J} \mathrm{Am}$ Coll Cardiol, 16(7), 1603-1607.

11. Feinstein, S. B., Cheirif, J., Ten Cate, F. J., Silverman, P. R., Heidenreich, P. A., Dick, C., Desir, R. M., Armstrong, W. F., Quinones, M. A., \& Shah, P. M. (1990). Safety and efficacy of a new transpulmonary ultrasound contrast agent: initial multicenter clinical results. $\mathrm{J}$ Am Coll Cardiol, 16(2), 316-324.

12. Schlief, R., Staks, T., Mahler, M., Rufer, M., Fritzsch, T., \& Seifert, W. (1990). Successful opacification of the left heart chambers on echocardiographic examination after intravenous injection of a new saccharide based contrast agent. Echocardiography, 7(1), 61-64.

13. Beppu, S., Matsuda, H., Shishido, T., Matsumura, M., \& Miyatake, K. (1997). Prolonged myocardial contrast echocardiography via peripheral venous administration of QW3600 injection (EchoGen): its efficacy and side effects. $J$ Am Soc Echocardiogr, 10(1), 11-24

14. GE Healthcare. (2008). OPTISON prescribing Information. Retrieved from http://www. amershamhealth-us.com/optison/

15. Skyba, D. M., Camarano, G., Goodman, N. C., Price, R. J., Skalak, T. C., \& Kaul, S. (1996). Hemodynamic characteristics, myocardial kinetics and microvascular rheology of FS069, a second-generation echocardiographic contrast agent capable of producing myocardial opacification from a venous injection. $\mathrm{J} \mathrm{Am} \mathrm{Coll}$
Cardiol, 28(5), 1292-1300. doi:10.1016/S07351097(96)00328-2

16. Luminity Summary of Product Characteristics. (2008). Retrieved from http://www.ema. europa.eu/docs/en GB/document library/ EPAR - Product Information/human/000654/ WC500045020.pdf

17. Definity-FDA prescribing information, side effects and uses (2001). Retrieved from https:// www.accessdata.fda.gov/drugsatfda docs/ label/2011/021064s011lbl.pdf

18. Sontum, P. C. (2008). Physicochemical characteristics of Sonazoid, a new contrast agent for ultrasound imaging. Ultrasound Med Biol, 34(5), 824-833. doi:10.1016/j. ultrasmedbio.2007.11.006

19. SonoVue INN-sulphur hexafluoride- European Medicines Agency (2014). Retrieved from www. ema.europa.eu

20. Dolan, M. S., Gala, S. S., Dodla, S., Abdelmoneim, S. S., Xie, F., Cloutier, D., Bierig, M., Mulvagh, S. L., Porter, T. R., \& Labovitz, A. J. (2009). Safety and efficacy of commercially available ultrasound contrast agents for rest and stress echocardiography a multicenter experience. $J$ Am Coll Cardiol, 53(1), 32-38. doi:10.1016/j. jacc.2008.08.066

21. Main, M. L., Goldman, J. H., \& Grayburn, P. A. (2007). Thinking outside the "box"-the ultrasound contrast controversy. J Am Coll Cardiol, 50(25), 2434-2437. doi:10.1016/j.jacc.2007.11.006

22. Main, M. L., Hibberd, M. G., Ryan, A., Lowe, T. J., Miller, P., \& Bhat, G. (2014). Acute mortality in critically ill patients undergoing echocardiography with or without an ultrasound contrast agent. JACC Cardiovasc Imaging, 7(1), 40-48. doi:10.1016/j.jcmg.2013.08.012.

23. EMEA. Scientific Discussion. European Medicines Agency. (2004). Retrieved from CPMP/0053/01.http://www.emea.europa.eu/ humandocs/human/epar/sonovue/sonovue.htm

24. Van Camp, G., Droogmans, S., \& Cosyns, B. (2007). Bio-effects of ultrasound contrast agents in daily clinical practice: fact or fiction? $\dagger$. European Heart Journal, 28(10), 1190-1192. doi:10.1093/eurheartj/ehm110

25. EMEA. http://www.emea.europa.eu/,document 
reference: Emea/cpmp/212/04

26. Porter, T. R., Abdelmoneim, S., Belcik, J. T., McCulloch, M. L., Mulvagh, S. L., Olson, J. J., Porcelli, C., Tsutsui, J. M., \& Wei, K. (2014). Guidelines for the cardiac sonographer in the performance of contrast echocardiography: a focused update from the American Society of Echocardiography. $J$ Am Soc Echocardiogr, 27(8), 797-810. doi:10.1016/j.echo.2014.05.011

27. Cosyns, B., Pasquet, A., Van Camp, G., Hernot, S., Droogmans, S., Unger, P., De Sutter, J., Lancellotti, P., \& Belgian Working Group on Non-Invasive Cardiac, I. (2009). Safety of ultrasound contrast agents: "Primum non nocere"? Acta Cardiol, 64(4), 547-552. doi:10.2143/AC.64.4.2041622

28. Schinkel, A. F., Kaspar, M., \& Staub, D. (2015) Contrast-enhanced ultrasound: clinical applications in patients with atherosclerosis. Int $J$ Cardiovasc Imaging. Advance online publication.

29. Amyot, R., Morales, M. A., \& Rovai, D. (2000). Contrast echocardiography for myocardial perfusion imaging using intravenous agents: progress and promises. Eur J Echocardiogr, 1(4), 233-243. doi:10.1053/euje.2000.0036

30. Abdelmoneim, S. S., Bernier, M., Scott, C. G., Dhoble, A., Ness, S. A., Hagen, M. E., Moir, S., McCully, R. B., Pellikka, P. A., \& Mulvagh, S. L. (2009). Safety of contrast agent use during stress echocardiography: a 4-year experience from a single-center cohort study of 26,774 patients. JACC Cardiovasc Imaging, 2(9), 1048-1056. doi:10.1016/j.jcmg.2009.03.020

31. Malhotra, V., Nwogu, J., Bondmass, M. D., Bean, M., Bieniarz, T., Tertell, M., Conliss, M., \& Devries, S. (2000). Is the technically limited echocardiographic study an endangered species? endocardial border definition with native tissue harmonic imaging and Optison contrast: a review of 200 cases. J Am Soc Echocardiogr, 13(8), 771773.

32. Rainbird, A. J., Mulvagh, S. L., Oh, J. K., McCully, R. B., Klarich, K. W., Shub, C., Mahoney, D. W., \& Pellikka, P. A. (2001). Contrast dobutamine stress echocardiography: clinical practice assessment in 300 consecutive patients. J Am Soc Echocardiogr, 14(5), 378-385.

33. Shah, B. N., Chahal, N. S., Bhattacharyya, S., Li, W., Roussin, I., Khattar, R. S., \& Senior, R. (2014). The feasibility and clinical utility of myocardial contrast echocardiography in clinical practice: results from the incorporation of myocardial perfusion assessment into clinical testing with stress echocardiography study. $J$ Am Soc Echocardiogr, 27(5), 520-530. doi:10.1016/j. echo.2014.01.028 